\title{
Developing classification criteria for skin-predominant dermatomyositis: the Delphi process
}

J.S.S. Concha (iD), ${ }^{1,2}$ S. Pena, ${ }^{1,2}$ R. G. Gaffney (iD, ${ }^{1,2}$ B. Patel, ${ }^{1,2}$ M. Tarazi (iD) ${ }^{1,2}$ C. J. Kushner (iD), ${ }^{1,2}$ J.F. Merola, ${ }^{3}$ D. Fiorentino, ${ }^{4}$ J.P. Dutz, ${ }^{5}$ M. Goodfield, ${ }^{6}$ F. Nyberg, ${ }^{7}$ B. Volc-Platzer, ${ }^{8}$ M. Fujimoto, ${ }^{9}$ C.C. Ang, ${ }^{10}$, V.P. Werth, (iD ${ }^{1,2}$ and The Skin Myositis Delphi Group

${ }^{1}$ Michael J. Crescenz VAMC, Philadelphia, PA, U.S.A.

${ }^{2}$ Department of Dermatology, Perelman School of Medicine at the University of Pennsylvania, Philadelphia, PA, U.S.A.

${ }^{3}$ Department of Dermatology, Brigham and Women's Hospital and Harvard Medical School, Boston, MA, U.S.A.

${ }^{4}$ Department of Dermatology, Stanford University School of Medicine, Redwood City, CA, U.S.A.

${ }^{5}$ Department of Dermatology and Skin Science, University of British Columbia, Vancouver, BC, Canada

${ }^{6}$ Department of Dermatology, Leeds General Infirmary, Leeds, U.K.

${ }^{7}$ Karolinska Universitetssjukhuset, Stockholm, Sweden

${ }^{8}$ Department of Dermatology, Wiener Krankenanstaltenverbund, Vienna, Austria

${ }^{9}$ Department of Dermatology, Faculty of Medicine, University of Tsukuba, Tsukuba, Japan

${ }^{10}$ Department of Dermatology, Changi General Hospital, Singapore

Linked Comment: Fernandez. Br J Dermatol 2020; 182:274-275

\section{Correspondence}

Victoria P. Werth.

E-mail: werth@pennmedicine.upenn.edu

\section{Accepted for publication}

1 May 2019

\section{Funding sources}

This work was supported by the United States Department of Veterans Affairs (Veterans Health Administration, Office of Research and Development and Biomedical Laboratory Research and Development).

\section{Conflicts of interest}

None declared.

See Appendix S1 for the full list of coauthors of the Skin Myositis Delphi Group.

DOI 10.1111/bjd.18096

\section{Summary}

Background The European League Against Rheumatism/American College of Rheumatology classification criteria for inflammatory myopathies are able to classify patients with skin-predominant dermatomyositis (DM). However, approximately $25 \%$ of patients with skin-predominant DM do not meet two of the three hallmark skin signs and fail to meet the criteria.

Objectives To develop a set of skin-focused classification criteria that will distinguish cutaneous DM from mimickers and allow a more inclusive definition of skin-predominant disease.

Methods An extensive literature review was done to generate items for the Delphi process. Items were grouped into categories of distribution, morphology, symptoms, antibodies, histology and contextual factors. Using REDCap ${ }^{\mathrm{TM}}$, participants rated these items in terms of appropriateness and distinguishing ability from mimickers. The relevance score ranged from 1 to 100 , and the median score determined a rank-ordered list. A prespecified median score cut-off was decided by the steering committee and the participants. There was a pre-Delphi and two rounds of actual Delphi.

Results There were 50 participating dermatologists and rheumatologists from North America, South America, Europe and Asia. After a cut-off score of 70 during the first round, 37 of the initial 54 items were retained and carried over to the next round. The cut-off was raised to 80 during round two and a list of 25 items was generated.

Conclusions This project is a key step in the development of prospectively validated classification criteria that will create a more inclusive population of patients with DM for clinical research.

\section{What's already known about this topic?}

- Proper classification of patients with skin-predominant dermatomyositis (DM) is indispensable in the appropriate conduct of clinical/translational research in the field. 
- The only validated European League Against Rheumatism/American College of Rheumatology criteria for idiopathic inflammatory myopathies are able to classify skin-predominant DM. However, a quarter of amyopathic patients still fail the criteria and does not meet the disease classification.

\section{What does this study add?}

- A list of 25 potential criteria divided into categories of distribution, morphology, symptomatology, pathology and contextual factors has been generated after several rounds of consensus exercise among experts in the field of DM.

- This Delphi project is a prerequisite to the development of a validated classification criteria set for skin-predominant DM.

Dermatomyositis (DM) is a heterogeneous autoimmune inflammatory disease that can be challenging to classify, particularly in patients who present predominantly with cutaneous disease. ${ }^{1}$ Unfortunately, skin-predominant DM has historically been left out of several classification criteria schemata, ${ }^{2-4}$ which has resulted in improper classification of patients and a delay in appropriate treatment and screenings. ${ }^{5}$ In addition, there can be interdisciplinary challenges among rheumatologists, neurologists and dermatologists in classifying these patients given the nuances of skin findings in DM. ${ }^{5}$ It is not surprising that a retrospective study showed that $55 \cdot 6 \%$ of patients had an alternate diagnosis prior to ultimately being diagnosed as having DM, with a median time delay from presentation to diagnosis of about 15 months. ${ }^{1}$ Altogether, $37 \cdot 2 \%$ of those alternate diagnoses were either systemic lupus erythematosus or cutaneous lupus erythematosus (CLE). ${ }^{1}$ These findings have major implications, as misclassifying patients can result in substandard screening for DM-associated findings, such as lung disease or cancer, ${ }^{6}$ as well as exclusion of these patients from clinical trials or translational studies. ${ }^{5}$

In an effort to improve existing classification criteria, a multidisciplinary collaboration, named the International Myositis Classification Criteria Project, has produced new European League Against Rheumatism/American College of Rheumatology (EULAR/ACR) classification criteria for idiopathic inflammatory myopathies, including clinically amyopathic DM $(\mathrm{CADM}) .{ }^{7}$ The new EULAR/ACR criteria include three DMassociated skin manifestations: Göttron sign, Göttron papules and heliotrope rash. ${ }^{7}$ Although these new criteria have made enormous improvements to the existing classification dilemma for patients with cutaneous DM, a retrospective study found that in using the new EULAR/ACR criteria, approximately $26 \%$ of patients with CADM did not meet the minimum probability cut-off for classification with $\mathrm{CADM}^{8}$

A proposed list of classification criteria for DM based purely on skin findings was presented to the Medical Dermatology Society more than 20 years ago, but it has not been further validated for use in clinical research. ${ }^{9}$ Despite this, recent interest in DM research has led to the use of these criteria as inclusion criteria for patient enrolment in clinical studies. ${ }^{10,11}$
In order to allow a more inclusive classification of skin-predominant DM, an effort to develop skin-focused classification criteria was started by an international group of experts in the rheumatology/dermatology field. This classification criteria project specifically aims to identify skin findings in DM that differentiate it from potential mimickers. The primary goal is to establish inclusive cohorts for clinical research on novel treatments for the disease. In doing so, the project can also be a mechanism to uniformly agree upon (i) a lexicon for the primary and secondary skin changes of DM; (ii) a consensus classification for the disease-defining cutaneous changes of DM; and (iii) identification of a sufficient combination of hallmark DM skin changes that need to be present for a confident classification of skin-predominant DM.

\section{Materials and methods}

This work formally began in early 2016, shortly after the commencement of the discoid lupus erythematosus (DLE) criteria project, when there was an identified need to standardize criteria for rheumatological diseases with prominent cutaneous features. ${ }^{12,13}$ After numerous discussions with specialists from North America, Europe and Asia, a consensus approach to criteria development was used. This decision was guided by earlier work on the classification criteria for systemic sclerosis, which employed a similar approach. ${ }^{14}$ The Delphi process is a feedback-based method in which convergence on a certain goal is reached by anonymously collecting data from experts using a set of iterative surveys, presenting the consensus to the participants and revising the successive surveys based on previously gathered data (Fig. 1). ${ }^{15}$ The array of answers (criteria items) is reduced every round until the group reaches a uniform consensus (using a measure of central tendency) on a desired end point (set of potential classification criteria meeting the median threshold). ${ }^{15}$

\section{Item generation for the Delphi process}

Criteria development begins with generation of candidate items (Fig. 1). ${ }^{13}$ The project started with an extensive literature search 
on and evaluation of the existing criteria for DM. ${ }^{4}$ This step in criteria development has previously been described in detail. ${ }^{4}$ The literature review also involved previous extensive work on the DM lexicon for CADM. ${ }^{9,16}$ Basically, gaps in the available criteria were identified, which justified the need to develop skinfocused criteria for DM. ${ }^{4}$ Data gathered from this search guided the generation of items for inclusion in the Delphi exercise.

Alongside the literature search, the steering committee (composed of international expert clinicians and researchers in the field of rheumatological dermatology) invited colleagues from around the world to assist in the generation of items for inclusion in the Delphi exercise. There were 43 participants who were asked to list, in a private chat forum, findings they think are relevant in classifying cutaneous DM. Collection of items occurred twice (first and second week of October 2016) before the consolidation phase (last week of October 2016).

\section{Item reduction}

The elimination process was conducted via internet-based surveys (pre- and formal Delphi) and nominal group discussions that were held during international meetings. Descriptive characteristics of participating experts are provided in Table 1. A majority of the participants have specialized in the field of DM for more than a decade.

\section{Rules of the Delphi process}

\section{Pre-Delphi exercise}

Using the RedCAP software (Research Electronic Database Capture Vanderbilt University, Nashville, TN, U.S.A.), ${ }^{17}$ an independent coordinator created an electronic questionnaire. The coordinator sent the link to the questionnaire via e-mail to 38 expert stakeholders composed of dermatologists, adult and paediatric rheumatologists, and dermatopathologists (Table 1). Using a 100-point scoring system set in a Likert scale, the participants graded each item in the questionnaire according to how relevant they were in classifying patients with skin-predominant DM from other mimickers. They also rated definitions of DM eponyms/signs based on their suitability to describe such terminologies. The median score for each item was used to create a rank-ordered list. Items reached consensus for inclusion if their median score was $\geq 70$, following the initial cut-off of the DLE criteria project. ${ }^{13}$ Items with median scores falling below the prespecified cut-off were placed in the 'no consensus' category and were subject to further nominal discussions if they were to be retained in the first round of the Delphi process.

\section{First round of the Delphi process}

The technique and scoring scheme used were similar to those used in the pre-Delphi exercise. Items with median values $\geq$ 70 were considered 'discriminating criteria'; those with median values between 30 and 70 were placed under the 'no consensus' heading; and criteria with median values $\leq 30$ were considered to have 'no distinguishing ability' and were immediately eliminated from the list.

\section{Second round of the Delphi}

The median cut-off for 'discriminating criteria' that was used for this round was raised to 80 , to generate a more concise

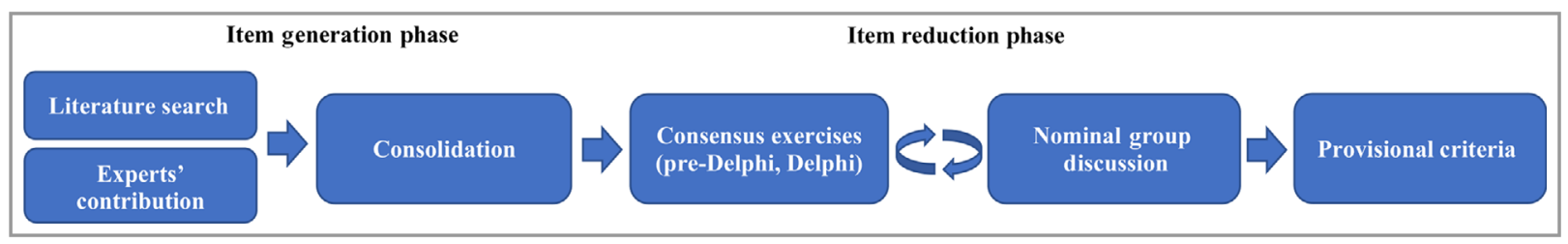

Fig 1. The process of criteria development began with item generation followed by item reduction.

Table 1 Demographics of participants

\begin{tabular}{|c|c|c|c|c|c|c|c|}
\hline & $\begin{array}{l}\text { Invitations } \\
\text { sent (n) }\end{array}$ & $\begin{array}{l}\text { Participants } \\
\text { (n) }\end{array}$ & $\begin{array}{l}\text { Response } \\
\text { rate }(\%)\end{array}$ & $\begin{array}{l}\text { Dermatologists } \\
\text { (n) }\end{array}$ & $\begin{array}{l}\text { Rheumatologists } \\
\text { (n) }\end{array}$ & $\begin{array}{l}\text { Rheumatologist- } \\
\text { dermatologist (n) }\end{array}$ & Continent of residence \\
\hline $\begin{array}{l}\text { Generation of items } \\
\text { for the Delphi exercise }\end{array}$ & - & 43 & & 37 & 6 & - & $\begin{array}{l}30 \text { North America, } \\
9 \text { Asia, } 4 \text { Europe }\end{array}$ \\
\hline Pre-Delphi & 38 & 32 & 86 & 28 & 4 & - & $\begin{array}{l}23 \text { North America, } \\
6 \text { Asia, } 3 \text { Europe }\end{array}$ \\
\hline Delphi round 1 & 38 & 50 & 88 & 43 & 3 & 4 & $\begin{array}{l}36 \text { North America, } \\
1 \text { South America, } \\
8 \text { Asia, } 5 \text { Europe }\end{array}$ \\
\hline Delphi round 2 & 51 & 50 & 98 & 42 & 4 & 4 & $\begin{array}{l}37 \text { North America, } \\
1 \text { South America, } \\
8 \text { Asia, } 4 \text { Europe }\end{array}$ \\
\hline
\end{tabular}


Table 2 Definitions of certain dermatomyositis (DM) items

\begin{tabular}{|c|c|}
\hline Poikiloderma & Poikiloderma includes features of hyperpigmentation, hypopigmentation, telangiectasias and epidermal atrophy \\
\hline $\begin{array}{l}\text { 'Red on white' pattern } \\
\text { of erythema }\end{array}$ & $\begin{array}{l}\text { This is a morphological finding that can occur anywhere on the body in patients with DM. It encompasses } \\
\text { several specific patterns. Most commonly, it includes an atrophic and/or ivory white background patch with } \\
\text { overlying telangiectatic erythematous macules in a follicular distribution. Of note, this patterning is distinct } \\
\text { from the poikiloderma that is also seen in dermatomyositis and from the poikiloderma due } \\
\text { to chronic sun damage }{ }^{18}\end{array}$ \\
\hline Mechanic's hands & $\begin{array}{l}\text { Changes seen on the fingers and palms (so-called Mechanic's hands) include a symmetric, nonpruritic, } \\
\text { hyperkeratotic, scaly eruption on the lateral surfaces of the digits, which can sometimes extend to the } \\
\text { palms and distal fingertips. Scaly linear papules in a similar distribution were recently described }{ }^{22}\end{array}$ \\
\hline Ovoid palatal erythema & $\begin{array}{l}\text { It has a characteristic bi-crescental pattern and is seen across the midline of the posterior hard palate at the } \\
\text { junction with the soft palate }{ }^{17}\end{array}$ \\
\hline Interface dermatitis & $\begin{array}{l}\text { Interface dermatitis is defined as a type of cutaneous inflammation in which the dermo-epidermal junction } \\
\text { is obscured. It is associated with vacuolar alteration at the dermoepidermal junction, necrosis of individual } \\
\text { keratinocytes and melanophages in the papillary dermis }{ }^{23}\end{array}$ \\
\hline
\end{tabular}

list of items that would make further validation of criteria more practical. This increase in the median cut-off was considered early on, between the first and second rounds, as guided by the preceding DLE criteria project. ${ }^{13}$ However, items with scores between 30 and 80 had no consensus, and those which fell $\leq 30$ were removed from the list.

\section{Nominal group discussions}

The primary goal of these discussions was to gather input on items that did not reach the cut-off threshold but that might still warrant inclusion in the next round of the Delphi, based on their potential to distinguish DM from mimickers. The international meetings that served as the venue for these discussions were the Rheumatologic Dermatology Society (RDS) meeting on 12 November 2016 in Washington, DC (for the results of the pre-Delphi exercise), the 2017 RDS meeting in San Diego (for round one) and the fourth International Conference on Cutaneous Lupus Erythematosus and Dermatomyositis (ICCLE) in May 2018 in Orlando, Florida (for round two). ${ }^{18}$ Members of the steering committee served as meeting facilitators.

\section{Results}

Overall, there was an excellent response to and completion rate of the Delphi surveys from rheumatologists and dermatologists from North America, South America, Europe and Asia (Table 1). As the pool of experts was limited, invitations for the subsequent surveys were sent to the participants of the previous Delphi exercise, as well as to several new referrals. For the last round of the Delphi process, the respondents were participants of the first round with one additional rheumatologist whose expertise is on criteria development.

\section{Pre-Delphi exercise}

An extensive list of DM features was generated after the literature search and online/face-to-face expert discussions (Table S1). Although this list was exhaustive, some items were redundant (e.g. Göttron papules and elbow/knee papules; Göttron sign and elbow/knee erythema/scale; heliotrope rash and eyelid oedema; V of the neck dyspigmentation and poikiloderma, etc.). There were 54 items retained after the pre-Delphi exercise.

The discussion following the pre-Delphi exercise was conducted to generate a more organized and manageable amount of data for the actual Delphi process. Participants of the RDS 2016 meeting resolved to group these items into the following categories: (i) distribution; (ii) morphology; (iii) patient symptoms; (iv) presence of myositis antibodies; (v) histopathology; and (vi) contextual factors (Table S1). The survey questionnaire to be used for the first round was redesigned to adhere to this categorization. The survey started with items that pertain to body areas affected by DM (eyelid, upper back, V of the neck, etc.) before proceeding into primary lesions seen in cutaneous DM (erythema, papules and/or plaques), and so forth. Because of ambiguity regarding the precise meaning of many popular terms, the participants voted to use clinical descriptions rather than eponyms or signs, with the use of pictures to increase precision (Table 2). For example, 'Göttron papule' and 'Göttron sign' were replaced with clinical descriptions [erythematous papules and/or plaques that are often flat topped, with or without scale, over the dorsal metacarpophalangeal (MCP) and/or interphalangeal (IP) joints; macular erythema over the dorsal MCP and/or IP joints, respectively]. Symptoms included were sensations of pruritus/burning/tingling, especially of the scalp; photosensitivity; and Raynaud's phenomenon. As all of the myositis-specific antibodies (MSA) scored $\geq 70$, this category was simply put as 'presence of any MSA' to take into consideration all the antibodies (anti-Mi-2, anti-Jo-1, anti-PL-7, anti-PL12, anti-EJ, anti-OJ, anti-KS, anti-155/140, anti-CADM140/ anti-MDA5, anti-NXP-2, anti-SAE). Histopathology retained 'interface dermatitis' and 'dermal mucin'; however, the steering committee added 'absence of spongiosis' to discriminate DM from an eczematous process. None of the direct immunofluorescent findings was carried over for further testing. Contextual factors included comorbidities such as interstitial lung disease by computed tomography, past or current malignancy, and the presence of objective muscle findings. 


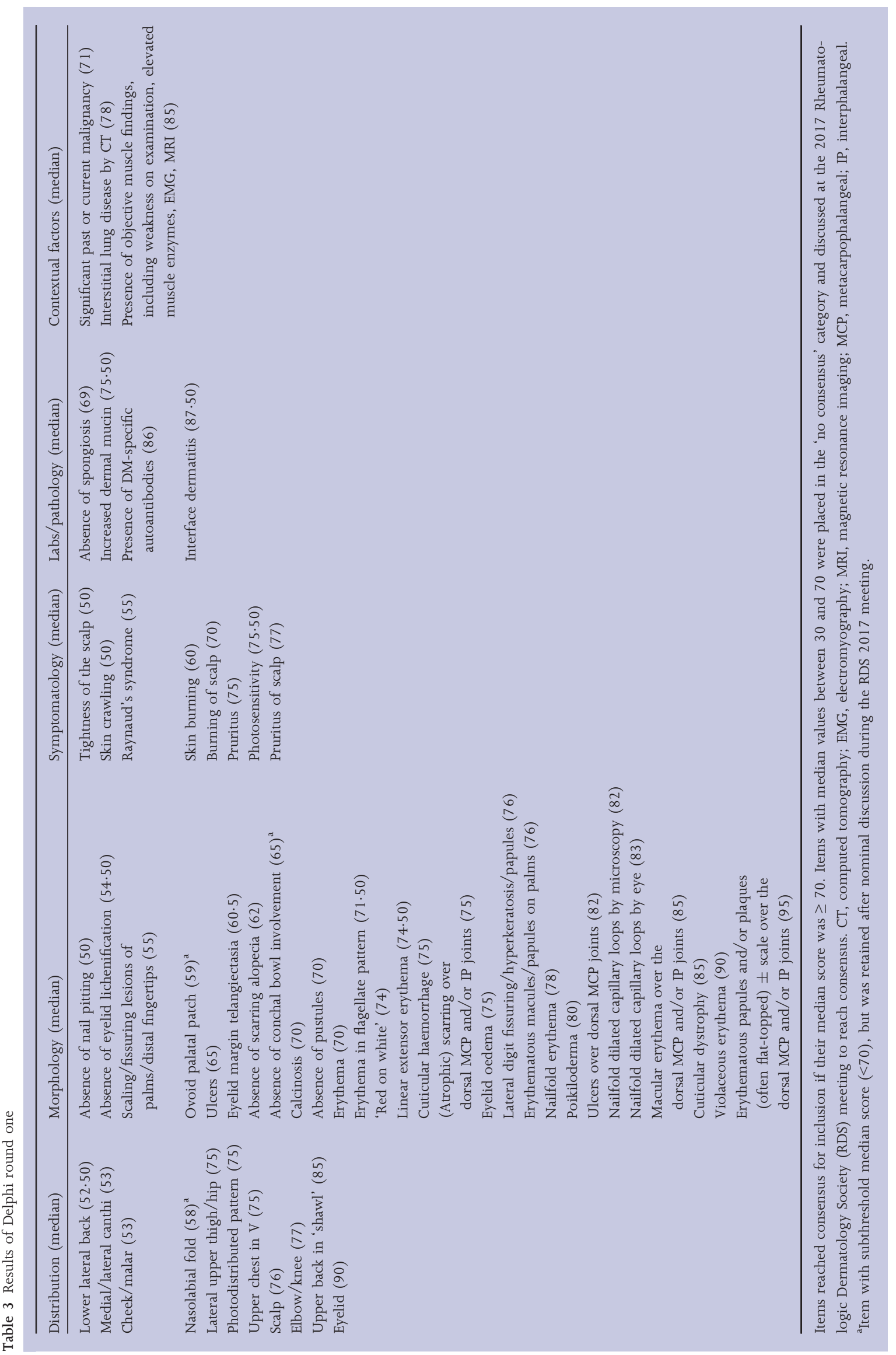


Table 4 Results of Delphi round two

\begin{tabular}{|c|c|c|c|c|}
\hline Distribution (median) & Morphology (median) & $\begin{array}{l}\text { Symptomatology } \\
\text { (median) }\end{array}$ & $\begin{array}{l}\text { Labs/pathology } \\
\text { (median) }\end{array}$ & $\begin{array}{l}\text { Contextual } \\
\text { factors (median) }\end{array}$ \\
\hline Nasolabial fold $(70)^{a}$ & $\begin{array}{l}\text { Absence of conchal bowl } \\
\text { involvement }(66.5)\end{array}$ & Burning of scalp $(71.5)$ & Dermal mucin (80) & Malignancy (72) \\
\hline Scalp $(75 \cdot 5)^{\mathrm{a}}$ & Absence of pustules (68) & Pruritus (75) & DM-specific & ILD on CT $(80)$ \\
\hline Lateral thigh $(75.5)^{\mathrm{a}}$ & Flagellate erythema (70) & Pruritus of scalp (80) & Myositis antibodies $(88.5)$ & Muscle weakness (85) \\
\hline Elbow, knee (80) & Calcinosis (70) & Photosensitivity (80) & Interface dermatitis $(90)$ & \\
\hline $\mathrm{V}$ of neck $(80)$ & Ovoid palatal erythema (70) & & & \\
\hline Shawl (83.5) & Joint scarring (75) & & & \\
\hline \multirow[t]{14}{*}{ Eyelid (85.5) } & 'Red on white' pattern $(75.5)$ & & & \\
\hline & Cuticular hemosiderin deposits (76) & & & \\
\hline & Ulcers MCP $(78.5)$ & & & \\
\hline & Poikiloderma (80) & & & \\
\hline & $\begin{array}{l}\text { Lateral digit fissuring/ } \\
\text { hyperkeratosis/papules ( } 80)\end{array}$ & & & \\
\hline & Linear extensor erythema $(80)$ & & & \\
\hline & Nailfold erythema (80) & & & \\
\hline & Eyelid oedema (80) & & & \\
\hline & Palmar macules and papules (81) & & & \\
\hline & Cuticular dystrophy (85) & & & \\
\hline & $\mathrm{MCP}, \mathrm{IP}$ joint macules (86) & & & \\
\hline & Nailfold capillary loops $(86 \cdot 5)$ & & & \\
\hline & Violaceous erythema (90) & & & \\
\hline & MCP, IP joint papules (90) & & & \\
\hline
\end{tabular}

Items reached consensus for inclusion if their median score was $\geq 80$. Items with median values between 30 and 80 were placed in the 'no consensus' category and discussed at the 2018 fourth International Conference on Cutaneous Lupus Erythematosus and Dermatomyositis (ICCLE) meeting to reach consensus. DM, dermatomyositis; ILD, interstitial lung disease; MCP, metacarpophalangeal; IP, interphalangeal. ${ }^{\mathrm{a}}$ Item with subthreshold median score $(<80)$ but was retained after nominal discussion at the fourth ICCLE meeting.

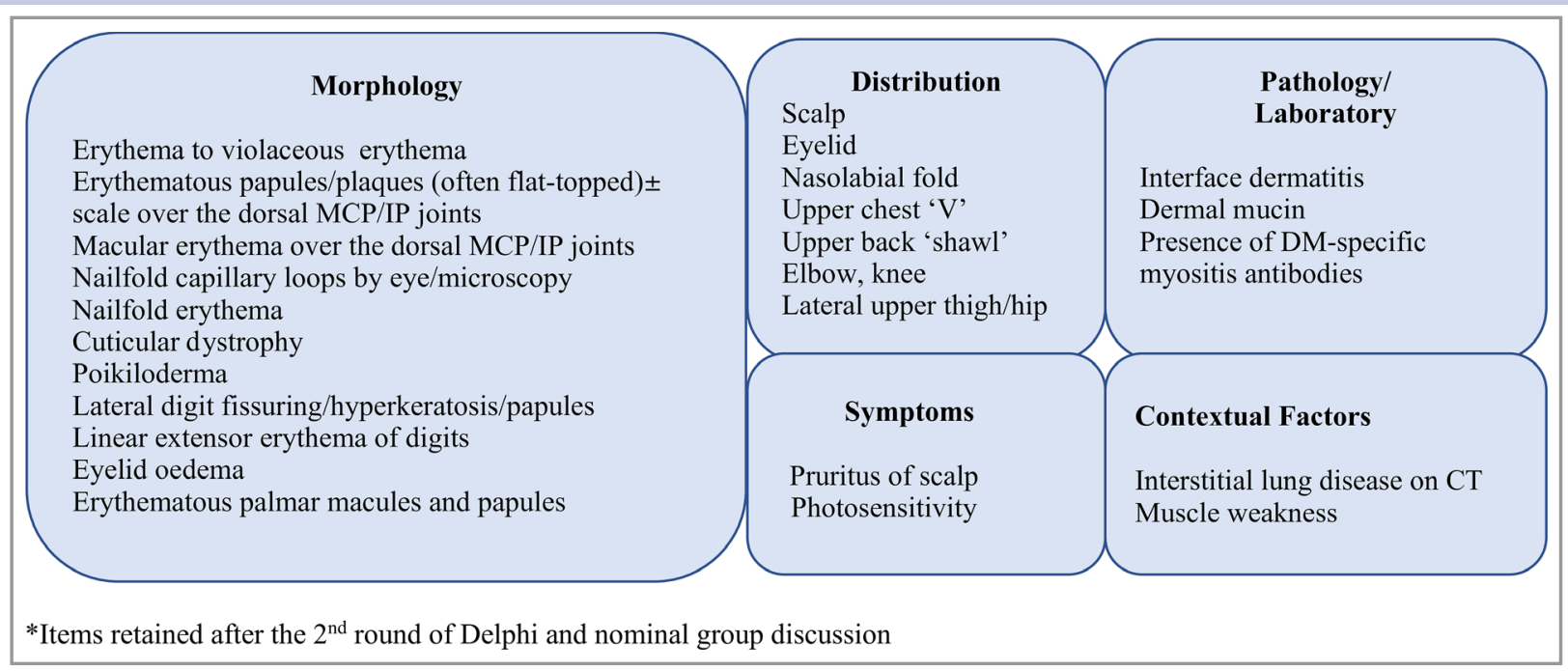

Fig 2. Potential* dermatomyositis (DM) classification criteria are stratified into the following categories: morphology, distribution, symptoms, pathology/laboratory and contextual factors. MCP, metacarpophalangeal; IP, interphalangeal; CT, computed tomography.

\section{First round}

The results of the first round of the Delphi were presented at the 2017 RDS meeting in San Diego (Table 3). A similar discussion was held to decide on items that did not reach consensus but that might still warrant inclusion in round two of the Delphi process. Following this, 37 items were retained.

\section{Second round}

The second round of the Delphi took place from January to April 2018. The respondents were participants of the first round with one additional rheumatologist whose expertise is on criteria development. There were 22 items retained after this round. However, at the fourth ICCLE meeting, ${ }^{18}$ there 
was agreement among the 93 participants to retain 'nasolabial fold' (70), 'scalp' (75.5) and 'lateral thigh' (75.5) because they were evaluated to have high discriminatory ability, especially in distinguishing DM from CLE.

\section{Discussion}

The results of these two rounds of Delphi represent the first consensus-based classification criteria for cutaneous DM (Fig. 2). The study group represents an international group of experts, each embodying multiple areas of expertise related to $\mathrm{DM}$, but largely driven by dermatologists. Overall, there was an excellent response rate from participants in all rounds. These potential criteria largely resemble prior diagnostic and classification criteria, which focused on the skin changes in DM. ${ }^{9}$ In contrast to previous criteria, eponymic signs have been replaced with more precise descriptive terms to better characterize cutaneous features of DM. Generation of this provisional list is only the first step in defining classification criteria for the cutaneous manifestations of DM. Further evaluation of items in a case-control, multicentre prospective study will enable us to assign a numerical weight to each item based on its specificity in a classification criteria set.

Items were divided into categories of distribution, morphology, symptomatology, pathology and contextual factors. Previous criteria required a skin biopsy consistent with DM to support a classification, ${ }^{9}$ and based on this consensus exercise, interface dermatitis and deposition of mucin in the deep dermis seem to be most consistent with the pathological features of cutaneous DM. In contrast to previous DM criteria, interstitial lung disease as a comorbidity, and the presence of a MSA, are new items that are considered critical clinical data when classifying patients with DM. The presence of muscle weakness was not intended primarily to characterize amyopathic DM; rather, it was used to reinforce the fact that if certain cutaneous items were concurrent with the evidence of clinical or subclinical muscle disease, these skin features are more likely consistent with DM. Conversely, if cutaneous features of DM are present, then it should prompt a clinician to search for underlying muscle involvement.

An inherent limitation of the Delphi process is the reliance on experts to decide which items to include (or exclude) based on their own experience, which may be biased according to their individual practice setting and prior medical training. For example, eyelid erythema (also found in eczema) may have consistently scored high vs. lateral thigh and nasolabial fold involvement because the heliotrope rash is a classically cited lesion of DM and perhaps not due to its high distinguishing ability from mimickers. Another possible limitation is the representation of experts, which might have affected the generalizability of the proposed criteria. However, the items on the provisional list were prevailing clinical features in the available literature on cutaneous DM and thus should be sufficiently characteristic of the disease and not entirely influenced by expert contribution. Although patients were not participants in this Delphi project, the steering committee and expert panel have worked on the development of patient assessment tools and quality-of-life indices in DM, which included systematic input from patients, and are therefore knowledgeable in the disease symptomatology of DM. ${ }^{19}$

In an attempt to produce criteria with high distinguishing power, the progressive increase in the cut-off score might have eliminated potentially DM-specific items from the list of potential criteria; however, no previous effort has ever been made to test the individual sensitivity/specificity of each criterion in DM, so a previous comparison cannot be done. Reducing the items to a list of 25 criteria was important to generate a more feasible list for prospective validation.

The overall goal of this criteria project was to classify patients with cutaneous features of DM and no muscle weakness to have skin-predominant DM. These criteria are not meant to replace the latest EULAR/ACR criteria, which are already adequate in classifying amyopathic DM. If prospectively validated, these classification criteria may be used if there is a high index of suspicion of DM in a patient who does not meet the EULAR/ACR criteria for amyopathic DM, but displays DM-specific skin changes in addition to Göttron papules, Göttron sign or heliotrope rash. They can also be used as standalone if, at the outset, the presentation is predominantly cutaneous. Therefore, they can be considered complementary to the existing EULAR/ACR criteria for DM.

In conclusion, a list of 25 potential criteria divided into categories of distribution, morphology, symptomatology, pathology and contextual factors has been a result of several rounds of consensus among experts in the DM field. These potential criteria do not represent a minimal set to classify cutaneous $\mathrm{DM}$, but will be subjected to further validation in prospective case-control testing to determine an item-specific probability score that will allow flexibility in combining criteria to sufficiently classify DM skin disease.

\section{References}

1 Da Silva DM, Patel B, Werth VP. Dermatomyositis: a diagnostic dilemma. J Am Acad Dermatol 2018; 79:371-3.

2 Lundberg IE, Miller FW, Tjarnlund A et al. Diagnosis and classification of idiopathic inflammatory myopathies. J Intern Med 2016; 280:39-51.

3 Lundberg IE, de Visser M, Werth VP. Classification of myositis. Nat Rev Rheumatol 2018; 14:269-78.

4 Concha J, Tarazi M, Kushner C et al. The diagnosis and classification of amyopathic dermatomyositis: a historical review and assessment of existing criteria. Br J Dermatol 2019; 180:1001-8.

5 Ghazi E, Sontheimer RD, Werth VP. The importance of including amyopathic dermatomyositis in the idiopathic inflammatory myositis spectrum. Clin Exp Rheumatol 2013; 31:128-34.

6 Iaccarino L, Ghirardello A, Bettio S et al. The clinical features, diagnosis and classification of dermatomyositis. J Autoimmun 2014; 4849: 122-7.

7 Lundberg IE, Tjarnlund A, Bottai M et al. 2017 European League Against Rheumatism/American College of Rheumatology classification criteria for adult and juvenile idiopathic inflammatory myopathies and their major subgroups. Arthritis Rheumatol 2017; 69:2271-82. 
8 Patel B, Khan N, Werth VP. Applicability of EULAR/ACR classification criteria for dermatomyositis to amyopathic disease. J Am Acad Dermatol 2018; 79:77-83.e1.

9 Sontheimer RD. Dermatomyositis: an overview of recent progress with emphasis on dermatologic aspects. Dermatol Clin 2002; 20:387-408.

10 Werth VP, Hejazi E, Pena SM et al. FRI0470 A phase 2 study of safety and efficacy of lenabasum (JBT-101), a cannabinoid receptor type 2 agonist, in refractory skin-predominant dermatomyositis. Ann Rheum Dis 2018; 77:763-4.

11 Werth VP, Patel B, Concha J et al. SAT0512 Safety and efficacy of lenabasum in refractory skin-predominant dermatomyositis subjects treated in an open label extension of trial jbt101-dm-001. Ann Rheum Dis 2018; 77 (Suppl. 2).

12 Elman SA, Nyberg F, Furukawa F et al. Developing classification criteria for discoid lupus erythematosus: an update from the World Congress of Dermatology 2015 meeting. Int J Womens Dermatol 2016; 2:44-5.

13 Elman SA, Joyce C, Nyberg F et al. Development of classification criteria for discoid lupus erythematosus: results of a Delphi exercise. J Am Acad Dermatol 2017; 77:261-7.

14 Van Den Hoogen F, Khanna D, Fransen J et al. 2013 classification criteria for systemic sclerosis: an American College of Rheumatology/European League Against Rheumatism collaborative initiative. Arthritis Rheum 2013; 65:2737-47.

15 Trevelyan EG, Robinson N. Delphi methodology in health research: how to do it? Eur J Integr Med 2015; 7:423-8.
16 Euwer RL, Sontheimer RD. Amyopathic dermatomyositis (dermatomyositis sine myositis): presentation of six new cases and review of the literature. J Am Acad Dermat 1991; 24:959-66.

17 Harris PA, Taylor R, Thielke R et al. Research electronic data capture (REDCap) - a metadata-driven methodology and workflow process for providing translational research informatics support. J Biomed Inform 2009; 42:377-81.

18 Concha JSS, Patsatsi A, Marshak-Rothstein A et al. Advances in cutaneous lupus erythematosus and dermatomyositis: a report from the 4th International Conference on Cutaneous Lupus Erythematosus - an ongoing need for international consensus and collaborations. J Invest Dermatol 2019; 139:270-6.

19 Goreshi R, Chock M, Foering $\mathrm{K}$ et al. Quality of life in dermatomyositis. J Am Acad Dermatol 2011; 65:1107-16.

\section{Supporting Information}

Additional Supporting Information may be found in the online version of this article at the publisher's website:

Appendix S1 Other co-authors of the Skin Myositis Delphi Group.

Table S1 Items collected and tested at the pre-Delphi stage, including definitions of selected dermatomyositis eponyms/ signs. 